\title{
Application of Automatic Data Collection Technology in Construc- tion Site Management
}

\author{
Henan Four Construction Co., Ltd. Kaifeng 475000, Henan
}

\section{Introduction}

In general, real-time effective construction site data collection has an important impact on building management. Traditional data collection technology requires a lot of manpower, financial resources, time, etc., site monitoring staff often need to spend nearly half of the time to collect and process data information, and manual data collection of staff have some experience requirements. However, complex and cumbersome manual data collection often lead to incomplete information or data errors, resulting in decision-making mistakes, inefficient and other issues. Advanced automation technology and information technology to promote the construction site to achieve efficient management. With the help of data automatic acquisition technology to track and locate the project object, and timely transmission and processing of data information, and then to the site monitoring personnel to transfer the specific situation of the construction site, so the construction site for effective monitoring and management.

\section{Automatic data acquisition technology for construction site}

Construction site information collection real-time, objectivity, comprehensiveness of the construction of the monitoring and management has an important role (see Figure 1). In addition, the collection of building construction information for the construction of monitoring and maintenance to create a system of information platform.1.1 Automatic identification technology

\section{(1) Bar code technology}

Barcode is a lot of spaced vertical bars, empty and corresponding characters that contain labels that contain some specific information. The technology was produced in the late 1940s, has now been widely used, such as large shopping centers, convenience stores and so on with its product management, which is a more applicable, economical automatic identification technology. At the same time with the mobile phone, handheld computers and other mobile devices and the emergence of the development of bar code technology more and more widely.

\section{(2) RFID technology}

RFID technology, that is, radio frequency identification technology, with radio frequency scanning to identify object objects and related information, completely change the manual mode of operation, suitable for some harsh construction site. The technical advantages of performance: remote scanning data information; storage capacity; information automatically updated; strong adaptability of the environment, and so on. However, the technology is still in the early stages of development, poor compatibility, high cost, but in its future development process, such problems will be solved.

\section{(3) Other technology}

In addition to the above two
Abstract: Data automatic acquisition technology is a new information technology in construction management. The use of this technology is to track and locate the project object, real-time transmission of data information, and through computer processing and analysis of data information, and then to the site monitoring personnel to transfer the specific situation of the construction site, so the construction site for effective monitoring and management. Based on the goal of strengthening the construction site management of the building, this paper classifies the data automatic acquisition technology from the functional use, and then studies the application of the data acquisition technology in the construction site management, hoping to improve the construction site management level reference function.

Key words: Automatic data acquisition technology; construction site management; application

Published online: 15th July, 2017

technologies, we usually come into contact with the automatic identification technology, including biometric technology, speech recognition technology, becauseof its use in the construction project and the use of a small range, here is not described in detail. 
1.2 Tracking and positioning technology

(1) GPS-outdoor tracking and positioning technology

The so-called GPS, the global positioning navigation system, can be located at any time, accurately locate the location. The system consists of ground control, space and user three parts, without the need for any sensor, through the satellite to receive or send signals to obtain the target location of the multi-dimensional positioning data. In addition, it is not affected by the environment and can work all day.

(2) Indoor tracking and positioning technology

The GPS technology for the line of sight outside the target positioning accuracy is low, and the loss of indoor satellite consumption. For example, for tunnel construction, GPS is difficult to ensure the accuracy of positioning. The emergence of short-range wireless communication technology, an effective solution to the problem of complex GPS applications for the construction site management to provide technical support.

\subsection{Image acquisition technology}

\section{(1) Video technology}

The so-called video surveillance that is, video surveillance, with the camera to record the scene, and then sent to the remote transmission system through the transmission medium, and install the lens rotation auxiliary equipment, all-round record of the scene. The technology to achieve the image and sound synchronization, real-time record construction site situation, reduce staff management pressure, improve the level of on-site management.

\section{(2) Laser scanning technology}

Laser scanning technology is one of the non-contact monitoring methods. The information acquired by laser scanning is objective and accurate, and it shows the characteristics and changes of the object objectively. Therefore, laser scanning technology is an important way to monitor spatial data.

1.4 Induction monitoring technolo- gy

Sensing device is the equipment that can sense the simultaneous conversion of the measurement object into an output signal. In the context of the rapid development of information technology, wireless monitoring needs to promote the induction monitoring technology. It is used to sense the monitoring information of each node of the sensing device. Each node generally includes small CPU, power supply, interface and other components, which can monitor the environmental information such as output temperature and air humidity.

\section{Application of Automatic Data Acquisition Technology in Construc- tion Site Management}

So far, the domestic construction management is still taking artificial data acquisition technology, and the technical information collection error, time-consuming, high cost, and data information is not timely increase the difficulty of monitoring the project management staff. Although the current automated data acquisition technology is still

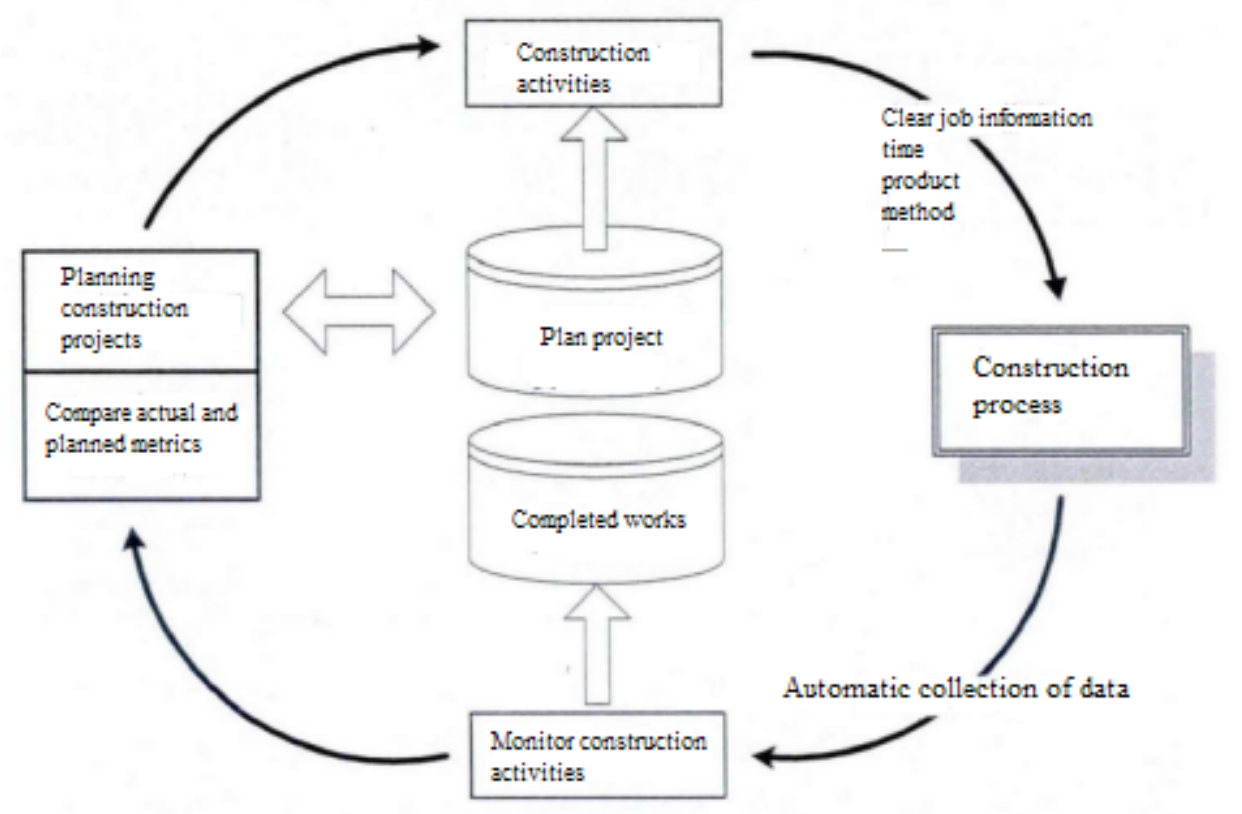

Figure 1 Construction process flow chart 
in the early stages of development, but its specific scope of use is also gradually expanded, mainly for the following: resource allocation, productivity analysis, quality management, schedule control and safety monitoring.

\subsection{Resource allocation}

For the construction of construction links, the human, building materials, equipment and facilities such as the optimal allocation of resources is to reduce costs, safety monitoring of the most important. The application of automated data acquisition technology to the construction site resource allocation is as follows:

(1) Building materials planning, procurement, storage, use (see Figure 2). At present, the construction site management materials on the construction of the main statistical classification of materials is chaotic; procurement plan is not perfect; material storage is not standardized; material recycling does not meet the requirements. The effectiveness of building materials management directly affect the efficiency of work, construction costs, so attention to the construction of materials management on the construction site monitoring has an important role.

(2) Staff, building materials, equipment, tracking and positioning. The realism of information gathering in construction site is directly related to the effectiveness of management decision. For the construction site of the above resources, can automatically identify the object and store the relevant information, while ensuring the location of the object and the information on the visualization, help managers quickly find the required information. The tracking of the above resources to some extent reduce the cost of manpower, improve the management level.

\subsection{Productivity analysis}

Generally speaking, the impact of the construction site on the productivity factors, including the surrounding environment, personnel professional level, material management, equipment, excellent degree. According to the data show that only about $20 \%$ of the information from the management to the grassroots construction personnel, so lack of communication is an important reason for low productivity. In this regard, automated data acquisition technology to improve this situation has an important role. Compared with the traditional manual acquisition, the technology improves the speed of equipment search; reduces the dependence on the staff; to strengthen the level of material management; based on intelligent algorithms can quickly find the reasons leading to reduced productivity.

\subsection{Quality management}

According to the data analysis, found that the main reason for the impact of building quality is human factors, such as the professional level of staff and management level, but also may be affected by building materials and systems. Automated data acquisition technology in the application of quality management are: piling in the bottom of the installation of RFID tags, thus determine the piling depth; for all types of building materials, size, specifications, categories, etc. stored in the system database to ensure the rational use of materials; Build a completed 3D model, the detection of building errors and deficiencies. In addition, the automatic detection system composed of the sensing equipment cannot be neglected for the construction quality management, and the output information of the sensor nodes is switched at the same time through the sensor nodes.

\subsection{Progress control}

Progress control is one of the main elements of the successful completion of construction. When the construction schedule deviates from the original plan, through the effective progress control, take the relevant adjustment means to keep up with the original construction plan. At present, the biggest application of

\begin{tabular}{c|c|c}
\hline Function & Barcode Technology & RFID Technology \\
\hline Read the number once & One & Can be multiple \\
\hline Read speed & $\begin{array}{c}\text { To position the read position, it } \\
\text { cannot be moved to read slowly }\end{array}$ & Moveable, fast \\
\hline Read distance & About 50 centimeters & $\begin{array}{c}1-100 m \text { (depending on frequen- } \\
\text { cy) }\end{array}$ \\
\hline
\end{tabular}

Table 1 Bar code technology and RFID technology comparison 
automatic data acquisition technology in the progress control is the visual inspection system, that is, the time of detection object to understand the progress of construction projects. For the system, the user can log directly query, site supervisors can view the impact of site construction, and through the network to communicate and control the progress of the construction site.

\subsection{Security monitoring}

In fact, due to the low level of professional staff, lack of communication, construction is not standardized, security monitoring system is not perfect, easily lead to construction site safety incidents. At present, there are two main ways to strengthen the safety monitoring of the construction site: first, to strengthen the safety concept of the construction personnel through training and encouragement; and second, to construct temporary protection facilities or supervise the construction personnel with single protective equipment. However, the above two ways is difficult to dynamically monitor the construction site safety. Therefore, the use of tracking and positioning technology can keep abreast of the staff with or without non-standard operation, and issue a warning reminder; automatic identification and positioning technology can be used to locate the location of workers in close to dangerous areas, timely warning to prevent further closer; But also with the help of high-precision sensing equipment to detect lifts and other operating conditions, according to the information collected by the alarm prompts, automatic control of some dangerous operation of the construction equipment, so as to effectively strengthen the construction site safety monitoring.

\section{Conclusion}

In general, advanced automation technology and information technology integration to replace the traditional artificial technology, to promote the construction site efficient management objectives. Data automation acquisition technology in the construction site management application, in line with the new era of sustainable development concept, to enhance the utilization of construction companies; reduce construction costs; to strengthen the construction site management standards; to avoid the occurrence of security incidents. Thus, data automatic acquisition technology with automatic identification, positioning tracking, real-time transmission and

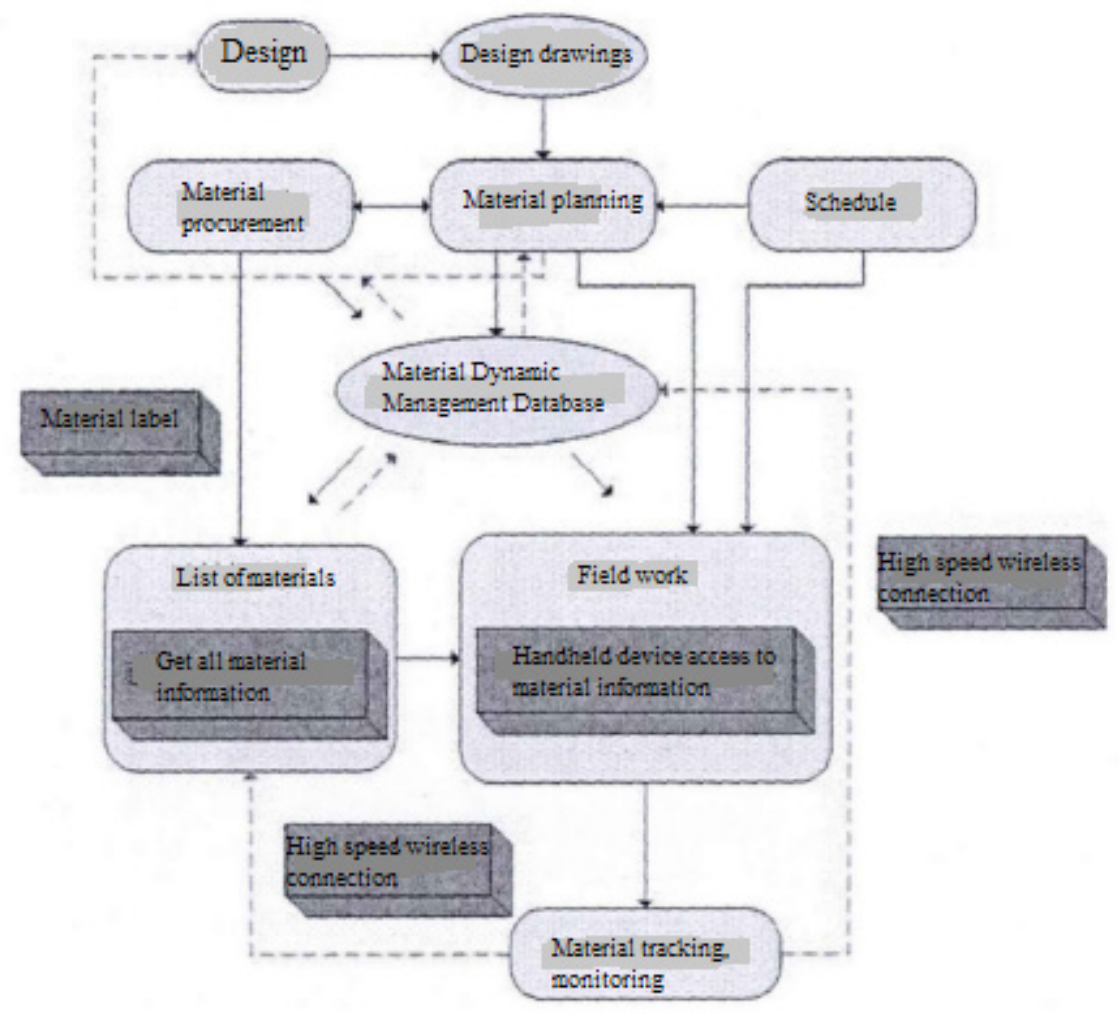

Figure 2 Schematic diagram of material management based on RFID technology 
other functions, in the construction site management in the use of a broad development prospects.

\section{References}

[1] Yang Shenghu. Bar Code Technology in the ERP System Application [J]. Petroleum and petrochemical materials procurement .2015 (02)

[2] Zhang Chao. Study on Mold Management System Based on Radio Frequency Identification Technology [J]. Aircraft Manufacturing Technology .2014 (05)

[3] Ji Jingjing. $A B C$ Classification Analysis in the Application of Construction Materials Management [J]. Construction Science and Technology .2014 (Z1)

[4] Tang Renzhong, Hu Luoke, Zhou Bang, Bai Ao. Study on Logistics Status of Workshop Products Based on Radio Frequency Identification Technology [J]. Computer Integrated Manufacturing System .2014 (01)

[5] Jinhoubing. Development of
Warehousing Material Management System Based On Wi-Fi Positioning [J]. Computer Knowledge and Technology .2013 (07)

[6] Zhang Jie, Guo Yanchen, Zhao Meng, Wang Peng. Information Management System under the Material Management [J]. Hebei enterprises .2013 (02)

[7] Ma Zhiliang, Zhang Dongdong, Qing Zhou, Liu Zhe, Yang Zhitian. Material Management System of Subway Engineering Construction Site Based on Mobile Terminal and Existing Information System [J]. Construction Technology .2012 (16)

[8] Jiang Shangting, Jin Yiren. Bar Code Technology and Its Application in Large Supermarket Management [J]. Journal of Anhui Electronic Information Vocational and Technical College.

[9] Jiang Fan. BIM and RFID Technology based on the Construction Project Safety Management Research [D]. Harbin Institute of Technology 2014
[10] Chen Xiang. Based on RFID Technology Outside the Scaffolding Security Real-Time Monitoring and Early Warning Method [D]. Harbin Institute of Technology 2012

[11] Ren Min. Based on the Identification and Evaluation of Dangerous Construction of the Construction Safety Early Warning System [D]. Xi'an University of Architecture and Technology 2008

[12] Hyun-Soo LeeKwang-Pyo LeeMoonseo Pa. RFID-Based Real-Time Locating System for Construction Safety Management. Journal of Computing in Civil Engineering.2012

[13] Kim, Changyoon, Kim, Hyoungkwan, Ryu, Jeoungpil, Kim, Changwan. Ubiquitous Sensor Network for Construction Material Monitoring. Journal of Construction Research.2011

[14] Seokho Chi, Carlos H.Caldas. Automated Object Identification Using Optical Video Cameras on Construction Sites.Computer-Aided Civil and Infrastructure Engineering.2011 